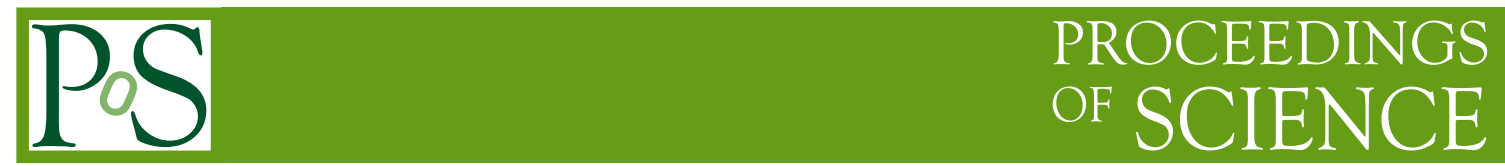

\title{
Polarisation of mJy radio sources
}

\author{
J. M. Stil* \\ Centre for Radio Astronomy \\ The University of Calgary \\ E-mail: stileras.ucalgary.ca
}

\section{A. R. Taylor}

Centre for Radio Astronomy

The University of Calgary

E-mail: russeras.ucalgary.ca

\section{Krause}

Max Planck Intitut für Radioastronomie, Bonn, Germany

E-mail: mkrause@mpifr-bonn.mpg.de

\section{R. Beck}

Max Planck Intitut für Radioastronomie, Bonn, Germany

E-mail: rbeck@mpifr-bonn.mpg.de

\begin{abstract}
Predictions of the number of faint polarised radio sources that can be detected by SKA pathfinder telescopes and the SKA depend on the polarisation properties of radio sources with a total flux density around $1 \mathrm{mJy}$. Total intensity source counts suggest a transition in the dominant population from AGN to galaxies around this flux density, and the properties of brighter radio sources may not be representative for this fainter population. We show that unresolved spiral galaxies can be highly polarised radio sources, up to $\sim 20 \%$ polarised at $4.8 \mathrm{GHz}$. This result is partly based on observations of nearby galaxies, including galaxies with significant deviations from axial symmetry and other peculiarities. A first analysis of polarised source counts divided into steep-spectrum AGN, flat-spectrum AGN and star forming galaxies is presented, including a prediction of polarised source counts to $\mu \mathrm{Jy}$ levels.
\end{abstract}

From Planets to Dark Energy: The Modern Radio Universe $1^{\text {st }}-5^{\text {th }}$ October 2007

The University of Manchester, United Kingdom

\footnotetext{
* Speaker.
} 

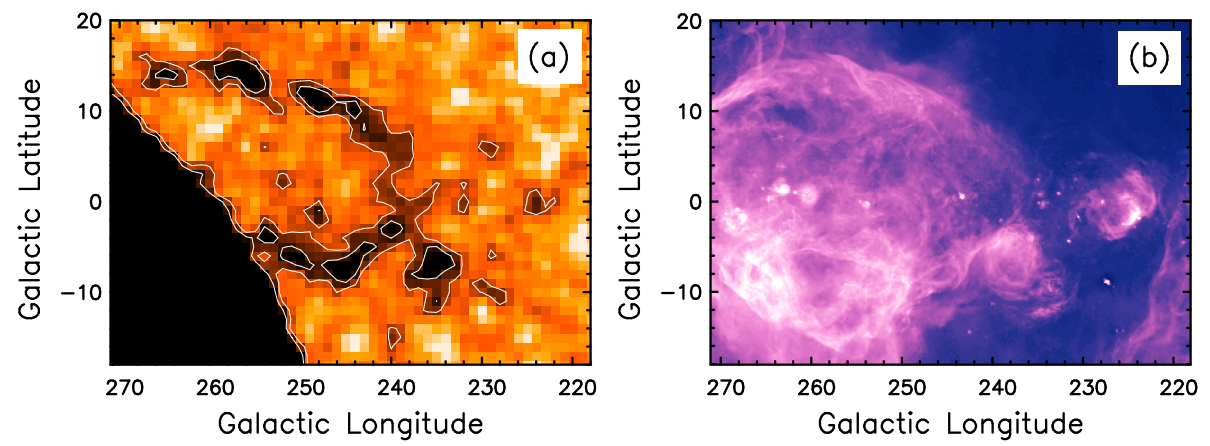

Figure 1: (a) Number density of polarised sources in the NVSS from [1]. Contours are drawn at 2.7 and 4.0 sources per square degree. The black area in the lower left corner is below the southern declination limit of the NVSS. (b) $\mathrm{H} \alpha$ intensity in the same region from the SHASSA survey [2].

\section{Science with polarised extragalactic sources}

SKA pathfinder projects currently under development aim to do $\mu \mathrm{Jy}$ sensitivity wide-area spectro-polarimetric surveys. These surveys will be capable of detecting many thousands of polarised sources, but the angular resolution $\lesssim 1^{\prime}$ will not be sufficient to resolve these sources. Nevertheless, many aspects of the SKA key science goal of the origin and evolution of cosmic magnetism can be studied with these deep polarisation surveys. By increasing the number density of polarised sources with observed rotation measure (the density of the rotation measure grid [3]), the magneto-ionic medium of the Galaxy and in nearby galaxies [ $₫$ ] can be mapped in much more detail than before. Figure 1 shows an example from [1] of what such studies may reveal. The left panel shows the number density of polarised sources in the NVSS [5] in the region of the Gum nebula. A strong deficiency below the mean background of 6.6 polarised sources per square degree is visible in a ring centered on $(l, b)=\left(-106^{\circ},+4^{\circ}\right)$ that coincides with an arc of $\mathrm{H} \alpha$ emission associated with the Gum nebula shown in Figure 1 (b). The deficiency in background polarised sources is the result of depolarisation resulting from a high rotation measure of material in a shell.

A polarimetric survey with ASKAP [6] may provide $\sim 20$ rotation measures per square degree over most of the sky. This number comes from a model-dependent extrapolation of the observed polarised source counts, $N(p)=1.25 p^{-2.5}$ for polarised flux density $20>p>0.7 \mathrm{mJy}$ [7], [7]. The question how faint the slope of the polarised source counts remains constant is crucial for the extrapolation to $\mu \mathrm{Jy}$ levels. Knowledge of the polarisation of radio sources of a few mJy total flux density is required for a realistic extrapolation. The first such extrapolation [3] was based on the polarisation properties of sources brighter than $80 \mathrm{mJy}$. These authors already noted that such an extrapolation would be a lower limit to the number of polarised sources if the fractional polarisation $\Pi_{0}$ of faint sources is higher, as found by a number of authors [7]-10]. Moreover, radio source counts suggest a population change around $1 \mathrm{mJy}$, so it is not clear that bright radio sources are representative for faint radio sources.

In addition to probing the magneto-ionic medium of the Milky Way, the intrinsic polarisation properties of extragalactic sources provide information on large-scale magnetic fields in distant galaxies. Observations of galaxies at high redshift will allow us to probe the cosmic evolution of magnetic fields. The resolution and sensitivity necessary to image the polarisation of normal 

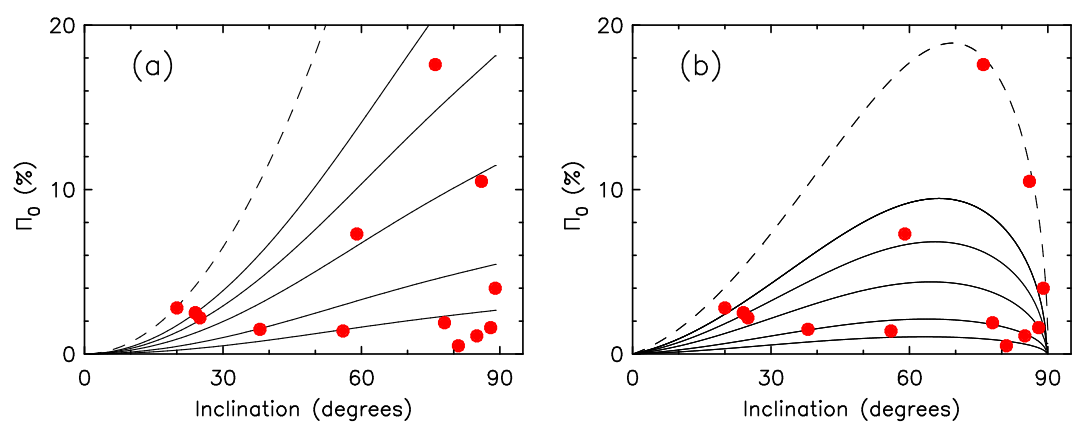

Figure 2: Integrated fractional polarisation $\Pi_{0}$ of nearby spiral galaxies (dots) with model curves for the expected integrated polarisation as a function of inclination without Faraday depolarisation (a) and with Faraday depolarisation (b). The curves represent models where the intensity of a face-on disk is 5, 10, 20, $30,40 \%$ (solid curves from bottom to top), and 70\% (dashed curve) polarised.

galaxies at high redshift will not be available before the SKA. For SKA pathfinders, we will be limited to information provided by integrated polarisation properties.

\section{Integrated polarisation of spiral galaxies}

Imaging of synchrotron emission from nearby spiral galaxies shows that most spiral galaxies have a regular azimuthal magnetic field in the plane of the disk [11]. If a spiral galaxy is observed face-on, the symmetry of the magnetic field implies no direction is favoured and all polarisation angles are represented equally by different portions of the disk. An unresolved axially symmetric face-on spiral galaxy will thus be an unpolarised radio source. For an inclined galaxy, the azimuthal magnetic field increasingly projects along the apparent major axis of the disk. This favours a plane of polarisation (B rotated by $90^{\circ}$ ) aligned with the minor axis. An unresolved inclined spiral galaxy is therefore expected to be polarised with plane of polarisation along the minor axis. This qualitative conclusion also applies to galaxies with spiral structure.

We investigated the integrated polarised emission from spiral galaxies by integrating archival Stokes Q and $\mathrm{U}$ images over the solid angle of the disk. Figure 2 shows the resulting integrated $\Pi_{0}$ at $4.8 \mathrm{GHz}$ for a sample of 13 nearby galaxies as a function of inclination [12]. The signal to noise ratio of the integrated fluxes is so high that polarisation bias is negligible. Some nearby galaxies would appear as highly polarised radio sources when observed as unresolved radio sources. The galaxies with the highest $\Pi_{0}$ in Figure 2 are M 31 (17.6\%), NGC 4565 (10.5\%), and M 81 (7.3\%).

Figure 2 also shows curves of predicted integrated $\Pi_{0}$ based on a simple axially symmetric model for the polarised intensity in the disk of a spiral galaxy with and without including the effects of Faraday depolarisation. Spiral arms, bars and vertical field components will be included in the future. The model evaluates magnetic field components perpendicular to and parallel to the line of sight and from this the polarised intensity and a simple prescription for Faraday depolarisation. The curves in Figure 2 form a single-parameter family where the parameter is the intrinsic fractional polarisation of the intensity from the disk as seen face on.

Galaxies at inclination $i \approx 65^{\circ}$ display the highest integrated polarisation. The cut off in $\Pi_{0}$ towards $i=90^{\circ}$ results from a strong increase in Faraday rotation because of a longer pathlength through the disk and larger line of sight components of the magnetic field, depending on position. 
The few galaxies with very high $\Pi_{0}$ seem to indicate that the simple treatment of Faraday depolarisation in our model may overestimate depolarisation effects in some galaxies. More detailed modeling is in progress. Approximately $47 \%$ of randomly oriented galaxies will have an inclination between $50^{\circ}$ and $80^{\circ}$, where the model curves predict a broad maximum. The probability density function of $\Pi_{0}$ for a randomly oriented set of spiral galaxies therefore has a maximum near the highest possible value of $\Pi_{0}$.

It is interesting to note that the integrated polarisation of spiral galaxies at low inclination is higher because of Faraday depolarisation. At low inclination, Faraday rotation increases mostly along the apparent major axis of the disk, where the line-of-sight component of the magnetic field increases linearly with inclination. The increased Faraday rotation and depolarisation reduce the axial symmetry of the polarised emission, which is the main source of depolarisation when integrating over the disk. This effect can be seen in the model curves of Figure 2 (a) and (b).

Integrated polarisation of spiral galaxies is a promising technique to study the large-scale magnetic fields in spiral galaxies for large samples. For an optically selected sample, the available information is $\Pi_{0}$ as a function of inclination, and the relation between the plane of polarisation and the position angle of the minor axis. If polarimetry at a second wavelength is available for a significant number of spiral galaxies, e.g. at $1.4 \mathrm{GHz}$ from the GALFACTS survey, the integrated polarisation of spiral galaxies allows a statistical study of large-scale asymmetry in the magnetic fields of galaxies, the occurrence of magnetic fields perpendicular to the disk, and the amount of Faraday depolarisation as a function of galaxy type.

Barred galaxies have the added complication of a possible magnetic field oriented along the bar. A sample of barred galaxies [13] displays similarly high integrated $\Pi_{0}$ [12]. Our conclusion that unresolved spiral galaxies can be highly polarised sources therefore includes barred galaxies. More detailed models will include bars and spiral structure in the near future.

\section{Models of polarised source populations}

The polarised source counts across the transition from AGN to star forming galaxies can be modeled by convolving total intensity counts for each population with the appropriate $\Pi_{0}$ distribution. We apply the $\Pi_{0}$ distribution of [3] to steep spectrum AGN $\left(\alpha>0.5, S_{v} \sim v^{-\alpha}\right)$, and the distribution of [7] to flat spectrum AGN. The $\Pi_{0}$ distribution for spiral galaxies was derived from the models in Section 2, and convolved with two model curves for the source counts of star forming galaxies. Many luminous starburst galaxies cannot be adequately represented by a spiral galaxy disk. We take the model source sounts for star bursts as an upper limit, and the model curve for a non-evolving local population as a lower limit to the number counts of star forming galaxies.

Figure 3 (a) shows model total-intensity source counts for different populations of radio sources, following [14]. Model polarised source counts obtained by convolving each of these curves with the appropriate $\Pi_{0}$ distribution are shown in Figure 3 (b), along with polarised source counts from the DRAO ELAIS N1 deep field [8]. The models in Figure 3 do not assume any variation of fractional polarisation with luminosity or flux density. The flatter slope of the normalised polarised source counts for $p<4$ mJy seems to be caused by a higher degree of polarisation of faint steep spectrum AGN. The excess mJy polarised sources in Figure 3 (b) were indeed identified with more highly polarised steep-spectrum AGNs by [8]. 

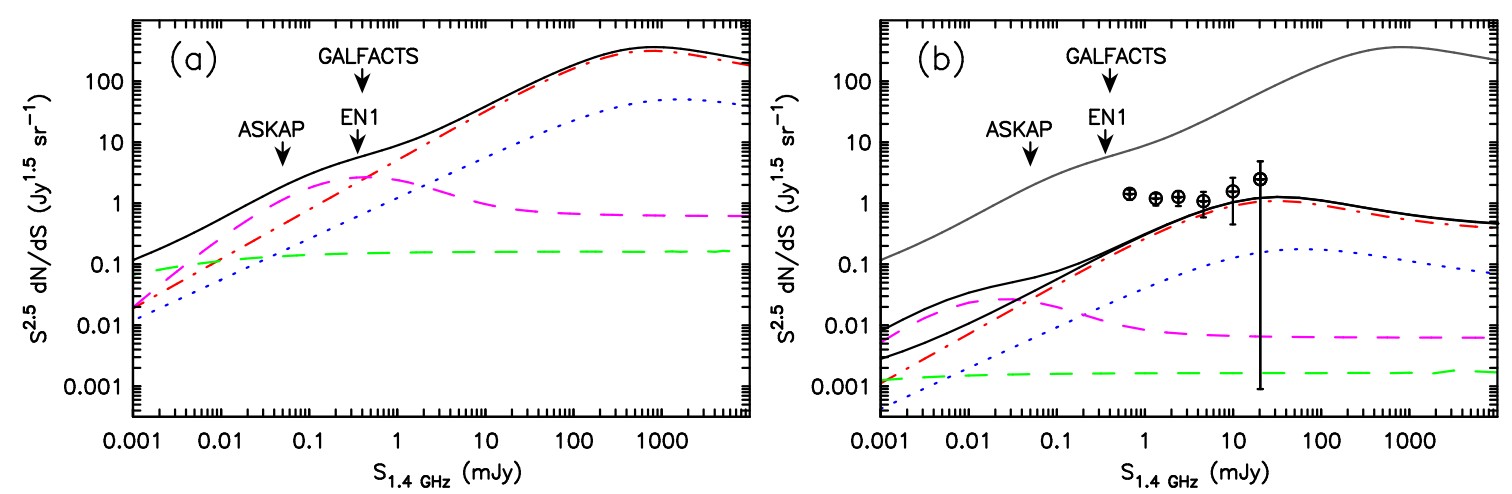

Figure 3: (a) Radio source counts calculated following [14]. Steep spectrum AGN: red dot-dashed curve. Flat spectrum AGN: blue dotted curve. Starburst galaxies: magenta dashed curve. Non-evolving spiral galaxies: green dashed curve. Black curve: total source counts. (b) Model polarised source counts obtained by convolving the curves in (a) with the $\Pi_{0}$ distributions described in the text. The data are observed polarised source counts from [8]. The $5 \sigma$ point source sensitivity of three polarisation surveys is indicated.

Why are faint steep-spectrum sources more highly polarised? It is possible that the large class of steep spectrum radio sources should be subdivided according to another parameter than spectral index. A low-luminosity slowly evolving sub-class of steep-spectrum sources (see the contribution by E. M. Sadler, this meeting) with a high $\Pi_{0}$ could be responsible for the discrepancy between the data and the models. A morphological distinction that is related to radio luminosity already exists in the Fanaroff \& Riley classification [15], but there is no evidence at this time that the faint polarised sources are indeed distant FR I sources.

\section{References}

[1] Stil, J. M., \& Taylor, A. R. 2007, ApJ, 663, L21

[2] Gaustad, J. E., McCullough, P. R., Rosing, W., \& Van Buren, D. 2001, PASP, 113, 1326

[3] Beck, R. and Gaensler, B. M. 2004, New Astronomy Reviews, 48, 1289

[4] Stepanov, R., Arshakian, T. G., Beck, R., Frick, P., \& Krause, M. 2007, arXiv: 0711.1267

[5] Condon, J. J., Cotton, W. D., Greisen, et al. 1998, AJ, 115, 1693

[6] Johnston et al. 2007, PASA, in press

[7] Tucci, M., Martínez-González, E., Toffolatti, L., et al. 2004, MNRAS, 349, 1267

[8] Taylor, A. R., Stil, J. M., Grant, J. K., et al. 2007, ApJ, 666, 201

[9] Mesa, D., Baccigalupi, C., De Zotti, G., et al. 2002, A\&A, 396, 463

[10] Sadler, E. M., Ricci, R., Ekers, R. D. et al. 2006, MNRAS, 371, 898

[11] Beck, R. 2005, in Cosmic Magnetic Fields, ed. R. Wielebinski \& R. Beck (Heidelberg:Springer), 41

[12] Stil, J. M., Krause, M. Beck, R. \& Taylor, A. R. 2007, in prep.

[13] Beck, R., Shoutenkov, V., Ehle, M., et al. 2002, A\&A, 391, 83

[14] Hopkins, A., Windhorst, R., Cram, L., \& Ekers, R. 2000, Experimental Astronomy, 10, 419

[15] Fananoff, B. L., \& Riley, J. M. 1974, MNRAS, 167, 31P 